\title{
SOCCER SONIFICATION: ENHANCING VIEWER EXPERIENCE
}

$$
\text { Richard Savery and Madhukesh Ayyagari }
$$

\author{
Georgia Tech Center for Music Technology \\ Georgia Institute of Technology \\ Atlanta, USA \\ rsavery3egatech.edu \\ madhukesh.ayyagari@gmail.com
}

\author{
Keenan May and Bruce Walker \\ Sonification Lab \\ Georgia Institute of Technology \\ Atlanta, USA \\ bruce.walker@psych.gatech.edu
kmayegatech.edu
}

\begin{abstract}
We present multiple approaches to soccer sonification, focusing on enhancing the experience for a general audience. For this work, we developed our own soccer data set through computer vision analysis of footage from a tactical overhead camera. This data-set included X, Y, coordinates for the ball and players throughout, as well as passes, steals and goals. After a divergent creation process, we developed four main methods of sports sonification for entertainment. For the Tempo Variation and Pitch Variation methods, tempo or pitch is operationalized to demonstrate ball and player movement data. The Key Moments method features only pass, steal and goal data, while the Musical Moments method takes existing music and attempts to align the track with important data points. Evaluation was done using a combination of qualitative focus groups and quantitative surveys, with 36 participants completing hour long sessions. Results indicated an overall preference for the Pitch Variation and Musical Moments methods, and revealed a robust trade-off between usability and enjoyability.
\end{abstract}

\section{INTRODUCTION}

Sports generate a wealth of data, including long-term statistics across games, seasons and careers, as well short-term analysis of player and ball movement during games. A large collection of research has developed across the last four decades focusing on using this data to improve physiology, psychology, and biomechanics[1]. In this paper we present and evaluate multiple approaches to soccer sonification, specifically geared towards entertainment for a general audience. Our goal is to use data to create an enhanced experience through increased perception of key events and complementary music.

\section{RELATED WORK}

Soccer data tracking and analysis is ubiquitous in professional soccer. These data are analyzed to help manage player fatigue [2], manage and identify long term trends such as the increased distance covered by players [3] and, crucially, to discover how these factors contribute to winning games[4]. Commercial 4.0 International License. The full terms of the License are available at http://creativecommons.org/licenses/by-nc/4.0
Sonification for sports and physical activity has been explored in many different research projects, although, to our knowledge, not for pure entertainment enhancement. Barrass et al. [5] compared six approaches to sonifying accelerometer data for nonspecific exercise, focusing on user enjoyment during exercise. Amongst these approaches, which were algorithmic music, sonification, weather metaphor, formants, musicification and streambased, the algorithmic music approach was shown to be the most popular, with participants noting it had a large amount of variety and was sensitive to their actions. Specific movements have been sonified to assist with physical activity such as squats [6], or to help predict future movements in sport [7], or to guide tactics [8]. Using sonification to optimize and improve athletic performance has been studied in the context of specific sports (elite sport rowing techniques [9]) as well as general techniques for real-time heart rate monitoring geared towards athletes [10]. Schaffert [11] presented results from a workshop on the use of real-time sonification to increase performance by athletes. Sports have also been sonified to allow visually impaired users to participate, such as sonified aerobics [12]. Conversely, many audio sports have been created, including an interactive soccer environment using only audio [13].

\section{SYSTEM OVERVIEW}

Our system is divided into three distinct components. First, the data are created through computer vision. They are then processed and mapped in MaxMSP, which in turn sends MIDI messages out to sample libraries and controls other parameters (such as tempo) in Logic. Figure 1 displays the system overview.

\subsection{Data Creation}

In our original sound design, creations we were able to use a data set collected directly by an established football club. However, for the purposes of evaluation and public sharing, we were required to use an external data set due to player data privacy laws. Some data sets exist containing soccer movements [14] although we were unable to find a data set that contained player movement and professional quality video. Professional clubs are understandably guarded about player and team data as it may lead to a competitive advantage. To create data we collected video from the tactical camera view of all rounds from the 2018 FIFA World Cup (see Figure 2). This footage allowed us to implement some relatively straightforward computer vision techniques to extract player and ball X,Y positions throughout the game. We started by analyzing the slight camera panning and movement using previously 


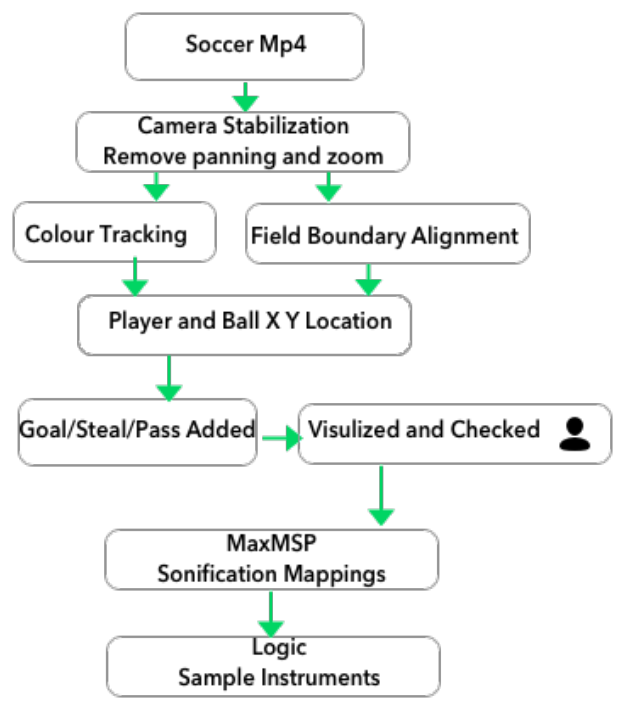

Figure 1: System Overview

created video analysis[15]. We then applied colour on the players and the ball, with the colours set by a human user before running the analysis. We were able to then bind the positions of the player by the boundaries of the field. These data were validated and, when necessary, corrected with the help of a Matlab visualization.

With $\mathrm{X}$ and $\mathrm{Y}$ data created for players and the ball, key soccer features were then generated, including possession, passes, steals, and goals. Possession was set by whichever player is closest to the ball. Goals were set whenever the ball passed through a set threshold. Passes were created when a ball travels a certain distance from a player, while steals were labelled when possession changed from one team to another. These higher level metrics are certainly not perfect, however, and required confirmation by a human.

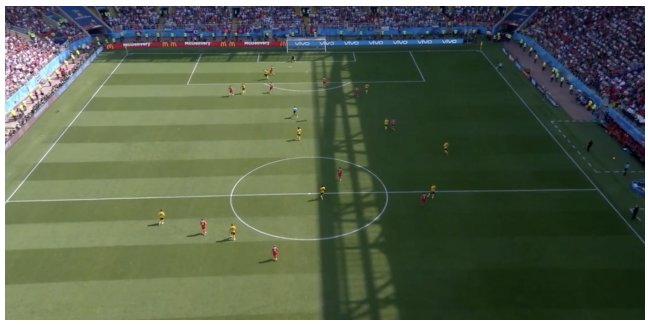

Figure 2: Tactical Camera View

\subsection{MaxMSP and Logic $X$}

After the data are created, they are loaded into MaxMSP${ }^{1}$ where mapping and processing occurs. In MaxMSP all calculations are done such as tempo mapping (linear or exponential), acceleration of the ball and players, and the distance between objects. From

\footnotetext{
${ }^{1}$ https://cycling74.com/
}

MaxMSP, MIDI pitches and control channels are sent to Logic where many sample instruments are controlled. Sample instruments include built-in Logic libraries, as well as libraries from 8dio, EastWest and Native Instruments.

\section{SONIFICATION MAPPING}

From the outset designing for entertainment and working with a specific football club guided us to certain grounding decisions. All sonifications were created from the viewpoint of one team, assuming the audience were supporting a set team. In early iterations we designed around a club's branding and nationality, however for evaluation and demos we moved to a generalized team sound. We also assume sounds would be used for clips no longer than three minutes long, allowing us to worry less about listening fatigue that could take place across a 90-minute game. Initial tests included possible representations of change throughout a season that would have allowed a players' sonic world to develop between games. The design went through an iterative process, creating many different approaches to the sound creation.

While we never aimed to directly replicate events shown through crowd noise we found this naturally occurred as plays built towards goals, or when possession changed. The design went through an iterative process creating many different approaches to the sound creation. After many divergent creations, we placed sonifications into four broad categories: pitch data mapping, tempo data mapping, musical moment alignment, and key moments.

\subsection{Game Clips}

To evaluate our different approaches, we used four clips from Belgium against Tunisia in Group $\mathrm{G}$ of the Fifa World Cup. We chose this game due to the variety of available plays and goals, with the final score $5-2$, to Belgium. We used Belgium as the supported team. Clip 1 (Goal 1) begins with the ball in Tunisia's possession, before a breakaway steal leads to a Belgium goal. Clip 2 (Goal 2) features a Tunisia goalie dropkick, followed by multiple Belgium passes eventually leading to a goal. Clip 3 (Penalty goal) is the shortest clip and features a goal scored after a penalty. Clip 4 (No goal) displays two shots on goal by Belgium with both blocked by the goalie, before the play disperses to midfield.

\subsection{Data Mapping}

Direct data mapping of ball and player distances from the goal, or to each other, became a key element of a two subgroups of our sonifications. These subgroups focused on mapping distances to either tempo, or pitch and the many possibilities that arise from this linkage. The following section describes guiding principles for each subgroup, followed by the evaluation where specific implementations are described.

\subsubsection{Tempo Mapping}

Through early internal testing we found operationalizing tempo as a measure of excitement was an effective technique. We created multiple demos featuring drum tracks generated using the author's previous system[16, 17]. This generative drum system allows control of rhythmic density of each cymbal, drum or percussive element individually. This category primarily focused on mapping the ball's distance from the goal to the tempo of the piece. 
Figure 3 displays the tempo curve (top line) followed by three lines of dynamic display, representing key player's distance from the ball. In this example each player had their own loop with the volume increasing as they became more or less involved in the play. In short term clips we found mapping dynamics ineffective as listeners were unable to quickly associate a sound with a player and therefore could not determine which dynamics corresponded to which player.

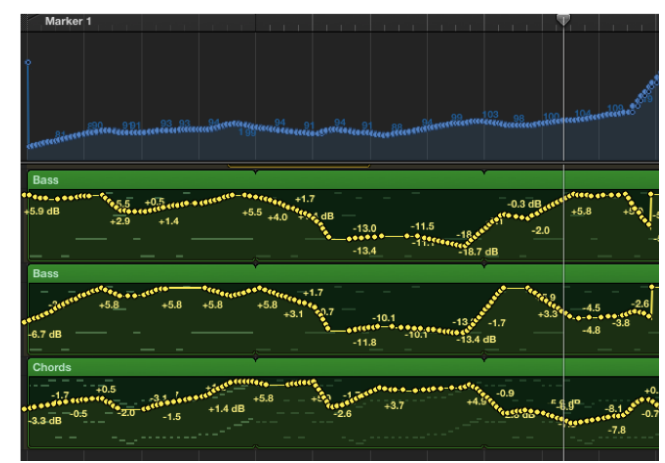

Figure 3: Dynamics Mapping

\subsubsection{Pitch Mapping}

Pitch variation utilized the data from the match in an almost identical way to tempo variation, however instead operationalized pitch as the driver for excitement. As in tempo mapping, certain elements of pitch were mapped to the distance of the ball from the goal.

\subsection{Key Moments}

For the key moment approach, we focused on only significant events and not player or ball positions on the field, including passes, steals, and goals. While these key events were also included in all the other sonifications this subgroup emphasized a deliberate focus on these interactions. They additionally allowed for enhanced listener focus on elements of these comments, such as the sonification of the ball speed during a pass.

\subsection{Musical Moment Alignment}

This category used existing pieces of music and aligned the most important moments in the soccer plays with the musically important features. This was done through intelligent identification of the important points in the data, and then working back through what was possible to align either through slight tempo variations, or cutting and reordering small sections of the composition. In general, the most important part of the clip was the goal, followed by a steal, and then other passes were aligned. This category is by far the least scalable as it requires human mapping, whereas all the other categories are automatically created by the pipeline described in Section 3.

\section{EVALUATION AND QUALITATIVE RESULTS}

\subsection{Process and Stimuli}

We ran evaluations with 36 participants, across 10 focus groups with 3 to 4 participants each. Participants were students in undergraduate classes, and were given partial class credit for their participation. Focus group sessions involved listening/viewing, plus discussion mixed with filling out an online survey form (using Qualtrics). Each session consisted of the following structure, and took an hour to complete.

Note that all stimuli are available at: http://richardsavery.com/soccersonification

1. Basic background questions about soccer playing and watching experience and regularity

2. Introduction to all four soccer clips used for evaluation without sound

3. A description of our goals in the project and an introduction to sonification

4. A tutorial track (tempo mapping for Goal 1) to demonstrate how sonification could be applied

5. For each of the subcategories (i.e. Key Moments):

- Viewing of each videos with discussion after

- Completing a Buzz Audio UX Scale [18]

- Writing out individual thoughts on the form

6. Final discussion and closing thoughts including consideration of all examples in comparison to each other

In the focus groups we aimed to ask about and analyze high level variables, such as the application of each category and understanding what did and did not work with each sonification approach. In general we aimed not to focus on low level parameters, such as the specific instrument sounds, as we were looking to develop a broader understanding of how sonification can enhance soccer and not focus too deeply on our own implementations of these sonification methods.

\subsection{Tempo Mapping}

\subsubsection{Stimuli}

For evaluation, we created four clips using tempo mapping. For Goal 1 we emphasized the tempo using a drum kit playing a groove throughout, with tempo linearly mapped between 80 beats-perminute (BPM) and 260 BPM to the ball's distance from the goal. An electric bass was mapped to the passes for the opposing team. The speed of the ball dictated whether 1,2, or 3 notes were played by the bass. For the supported team, an electric guitar was mapped to passes, steals, and goals. This clip was designed as a general tutorial for the participants in the evaluations described later.

For Goal 2, we used a 2 bar drum loop with a repeating bass line to demonstrate the tempo, in this case mapped exponentially between 40 BPM and 260 BPM to the distance from the goal. Passes, steals and goals were mapped with a chime synth, with pass length tied to note length. The penalty goal used only the drum kit, to demonstrate the contrast between relatively fixed positions, as the clip begins with the ball stationary. The penalty goal had a small range of $x, y$ positions, with the associated tempo ranging between 80BPM and $180 \mathrm{BPM}$. The non-goal used a new drum 
groove underneath, again exponentially mapped between 80 BPM and 260 BPM, combined with a different pass sound.

\subsubsection{Feedback}

In general, respondents found that having tempo mapped underneath added extra excitement and increased tension to the play. There was a consensus throughout all groups that the sounds amplified what is happening on field, with one noting that it was like listening to a more detailed version of the crowd. As to be expected, participants varied in their style preferences for the underlying groove. However, there was agreement that grooves featuring not just drums conveyed a clearer sense of ball position. The variation in contrast between exponential and linear mapping was noticed, although preferences were split between each category.

For the penalty goal only using drums, we heard repeatedly that drums do not convey much information, and that the contrast from a slow tempo to fast tempo with only drums wasn't particularly clear. Many participants noted that Tempo gives the idea of how fast the players are moving, with most arguing this as a positive; however two participants felt the slower sections made the play feel slower and less interesting. In addition some argued that these contrasts didn't always replicate the real situational intensity of the play. For the clips overall there was disagreement about whether the intensity level was correct or not, with some describing the music as intense for what would happen during the game, while others thought the music wasn't intense enough.

\subsection{Pitch Mapping}

\subsubsection{Stimuli}

Three evaluation tracks were created for pitch mapping. The first clip (sonifiying Goal 2) used an electric bass playing repeating eighth notes at 120 BPM. The pitches were then exponentially mapped to the ball's distance from the goal, the closer the ball to the goal the higher the pitch. Underneath the bass a generated drum track was created, with variations in density also mapped to this distance, although divided into 7 density levels. Passes, goals and steals were mapped using the same guitar sound used for goal 2 tempo mapping. The second pitch mapping example was created for the penalty goal and uses a bassoon and flute. Both instruments play eighth notes at 120 BPM. The bassoon's pitch is mapped to the ball's distance from goal. The flute's pitch is mapped to the shooting player's distance from the ball: as he approaches the ball the pitch rises, and then falls again as the ball travels away. The third pitch mapping track was created for the non goal clip. In this clip pitch changes were quantized per measure of music, with the average distance over that measure used to set the pitch.

\subsubsection{Feedback}

Overall, Pitch Mapping and Key Moments received the most positive qualitative feedback from participants. The clip created for Goal 2 was many participants' favorite track, with some labelling it like a song. Others described the track made them feel a good nervous, as it was like a car chase. They also noted that while tempo was good for excitement, pitch was generally easier to understand, and its mapping conveyed the ball's position in a clearer manner. Ultimately for the Goal 2 clip many participants agreed it was All encompassing of what you look for in the game. The second pitch clip created for the penalty goal was in general well received for its information content. For the pitch clip for the non goal all participants found the pitch hard to understand, due to only shifting pitch per measure. We believe this was all due to the lack of a tonic creating a guide for the pitch, so adjustments were very hard to distinguish for a general audience.

\subsection{Key Moments}

\subsubsection{Stimuli}

The first key moments example was for Goal 2, and used only a drum kit. This track featured changes in density, volume, and cymbals/parts of the drum kit to demonstrate when a key moment occurred. The second example was created for the penalty goal, with just the shot and goal sonified, through a change of musical tone. This was done by moving from a V chord to the I after the goal, with a change of groove. The final clip created for the non goal used solely piano, with each key moment sonified through mapping of pitch, volume, and note length. Actions between teams were differentiated by the octave of the piano, with a lower octave given to the opposing team. The note length was mapped to the duration of each pass. Passes from each team were sonified with a piano tremolo: over a minor chord for the opponent team, and over a major chord for the supported team.

\subsubsection{Feedback}

The clip for Goal 2 was generally disliked by participants, with many describing only drums as confusing, and Not expressing any information by itself, but supporting the story. The penalty goal was described as matching the joy of scoring a goal, with the music accurately capturing the mood and the euphoria of scoring a goal. The final key moments clip created for the non goal received many positive comments. Participants noted that the use of piano changed the perception of the ball, with it at times seeming lighter than in other clips. The use of silence in this clip received different interpretations, with some describing it as helpful to only emphasize important parts while others described the silence as distracting. Many participants said this clip was the clearest to follow, with significant differences between each teams' actions and a good portrayal of what was happening.

\subsection{Musical Moment Alignment}

\subsubsection{Stimuli}

Goal 1 used a carefully chosen collection of rock loops. The ending of the song lined up with the goal, while the bridge was able to line up with the steal in the play. The bridge also featured a crescendo, and rise in pitch that loosely corresponded to the ball's distance from the goal. Using slight variations in tempo several passes were also aligned with the pulse of the piece. For the second goal we used loops of a funk soul groove, with the ending of the piece synchronized to the ball entering the goal. Other alignments included saxophone and trumpet layers coming in and out at important moments of the play. The third musical moment alignment clip was created for the penalty goal. This clip lines up just when the goal is scored, combining suspended trumpet rubato line pre-goal, followed by a mariachi inspired groove after the goal. 


\subsubsection{Feedback}

Musical Moment Alignment was unsurprisingly described as the most musical, due to the fact standard pieces of music were used for the creations. As expected, participants also described that these examples were the least helpful in understanding the game; however, many noted it did support the play. One participant noted that the way the piece lined up showed that soccer itself is musical. Some participants described Goal 1 as making it harder to focus on the fine points of the play, even though they noted the sounds made the track more enjoyable. No participants noticed the horn lines syncing with passes for Goal 2, likely due to an inconsistent mapping. Overall, almost none of the participants found the approach to Goal 2 effective. The penalty goal for this category was by far the most polarizing clip used in the evaluation. Responses ranged from describing it as perfectly matching the tension of a penalty goal, followed by the joy of scoring, while others thought it drastically overplayed the clip. Participants did unanimously enjoy this section of clips, although commonly noted it made the clip feel like a highlight reel, and not like they were involved in the play itself.

\section{QUANTITATIVE RESULTS}

For quantitative analysis we used the BUZZ Audio User Experience Scale [18]. The BUZZ scale is comprised of eleven questions about the usability,usefulness and aesthetics of the sounds used in auditory displays and user interfaces. The BUZZ scale was designed to be applicable to a variety of different systems, as well as generalizable, allowing comparisons to be made across different systems. It is typically analyzed both by combining all eleven questions into one composite score, and by decomposition via factor analysis.

\subsection{BUZZ Composite Scores}

A Hyunh-Feldt repeated-measured ANOVA indicated that there was a significant effect of Sonification Method on BUZZ composite scores, $F(2.856,97.114)=5.344, p=.002, \eta_{p}^{2}=.136$. As shown in Figure 4 and Table 1, participants rated the Musical Moment and Pitch Mapping conditions more highly than the Key Moment condition. Additionally, a linear regression model revealed that an unweighted composite of the soccer experience and preference questions was not a significant predictor of BUZZ composite scores, nor did this item interact with Sonification Method.

\subsection{BUZZ Subscale Scores}

To identify factors within the BUZZ results, a principle factor analysis with Varimax rotation and Kaiser Normalization was conducted, as recommended by [18]. Items 2,3,8, and 9 loaded on a factor reflecting the enjoyment and appeal of the sounds, and items $1,4,5,7,10$, and 11 loaded on a factor reflecting ease of use. Those items were combined into an unweighted sum, to produce scores for those two factors. Analyses of those factors are recounted below.

\subsubsection{Enjoyment and Appeal}

A Hyunh-Feldt repeated-measured ANOVA indicated that there was a significant effect of Sonification Method on BUZZ Enjoyment and Appeal scores, $F(2.771,94.208)=21.474, p<.001$, $\eta_{p}^{2}=.387$. Table 2 and Figure 5 show that participants rated the Pitch Variation condition more highly than the other three conditions. Within those three conditions, the Key Moment condition was rated lower than the other two. This indicates that participants found the Pitch Variation Sonification Method most enjoyable and appealing to listen to.

\subsubsection{Ease of Use}

A Hyunh-Feldt repeated-measured ANOVA indicated that there was a significant effect of Sonification Method on BUZZ Ease of Use scores, $F(2.661,90.466)=10.232, p<.001, \eta_{\mathrm{p}}^{2}=.231$. Table 3 and Figure 6 show that participants rated the Musical Moment and Pitch Sonification conditions more highly in terms of ease of use. Notably, the Pitch Sonification was rated lower in terms of ease of use compared to Musical Moment.

\section{DISCUSSION}

Through evaluation we developed multiple takeaways. These focused on what worked as we expected, what surprised us, and what we could use for future developments.

\subsection{Point of Focus}

Different sonification methods significantly shifted the way participants watched the game and their point of focus. Depending on the sonification tactic employed, participants would focus on different aspects of the play. This included a change between micro and macro level aspects, with some sonification methods drawing listeners to focus less on the broader game and more on the movement. There is no clear answer to what is best to draw attention to, and particularly for entertainment this will vary between viewers.

\subsection{Managing Interpretation of Events}

The sonification of key moments drew several comments about the placement of the sound. For passes some participants noted that the event happened before the sound, while others commented the sound was too early. Attempting to sonify a sporting event ultimately forces many of these decisions to be made by the creators, with just soccer passes open to many interpretations and sonification methods.

\subsection{Supporting a Team}

Our approach to always have a single supported team was well received in these evaluations. With a data-driven approach this seems a logical choice to sustain, since either team could be automatically sonified. In general, though, participants also believed that many of our sonification methods could be used for either team, with approaches-to-goal being conveyed as suspenseful for both the defending and attacking team.

\subsection{The Use of Drums}

Overall the use of drums as a guiding feature in the sonifications was not effective. There was a general attitude that subtleties could not be distinguished as relating to moments in the soccer game. 


\begin{tabular}{||ccccc||}
\hline & Tempo & Key & Musical & Pitch \\
\hline Mean & 33.31 & 30.60 & 35.94 & 37.54 \\
\hline SE & 51.36 & 55.38 & 54.12 & 62.76 \\
\hline Differs From: & - & music,pitch & key & key \\
\hline
\end{tabular}

Table 1: BUZZ Composite Scores (out of 77) by Sonification Method

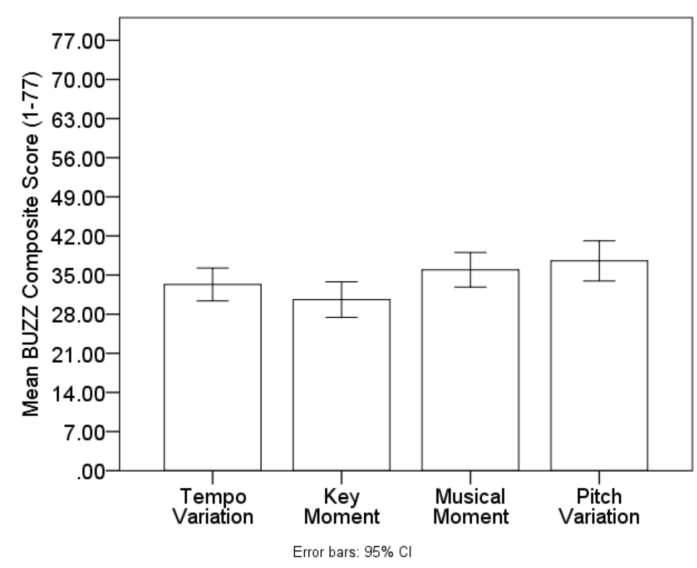

Figure 4: BUZZ Composite Scores (out of 77) by Sonification Method

\begin{tabular}{||ccccc||}
\hline & Tempo & Key & Musical & Pitch \\
\hline Mean & 12.54 & 10.74 & 8.71 & 15.57 \\
\hline SE & 20.16 & 24.60 & 21.24 & 31.62 \\
\hline Differs From: & music,pitch & pitch & $\begin{array}{c}\text { pitch } \\
\text { tempo }\end{array}$ & $\begin{array}{c}\text { tempo,key } \\
\text { music }\end{array}$ \\
\hline
\end{tabular}

Table 2: BUZZ Enjoyment and Appeal Scores (out of 21) by Sonification Method

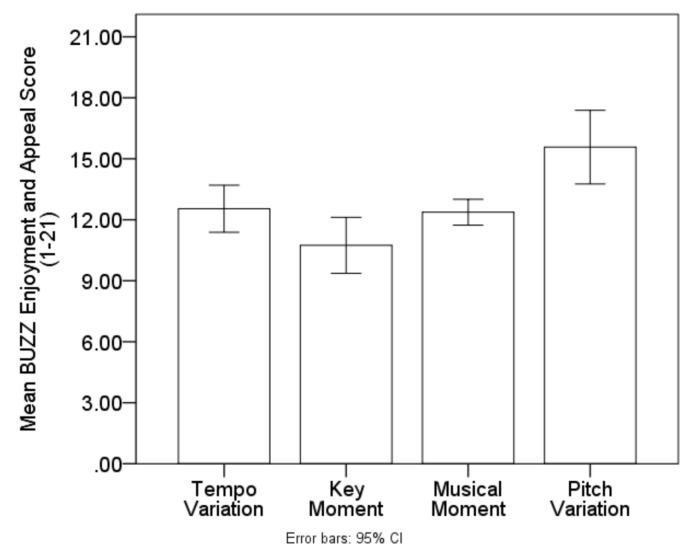

Figure 5: BUZZ Enjoyment and Appeal Scores (out of 21) by Sonification Method

\begin{tabular}{||ccccc||}
\hline & Tempo & Key & Musical & Pitch \\
\hline Mean & 21.60 & 19.86 & 27.23 & 21.97 \\
\hline SE & 19.38 & 39.72 & 47.58 & 44.10 \\
\hline Differs From: & music & music & tempo,key, pitch & music \\
\hline
\end{tabular}

Table 3: BUZZ Ease of Use Scores (out of 56) by Sonification Method

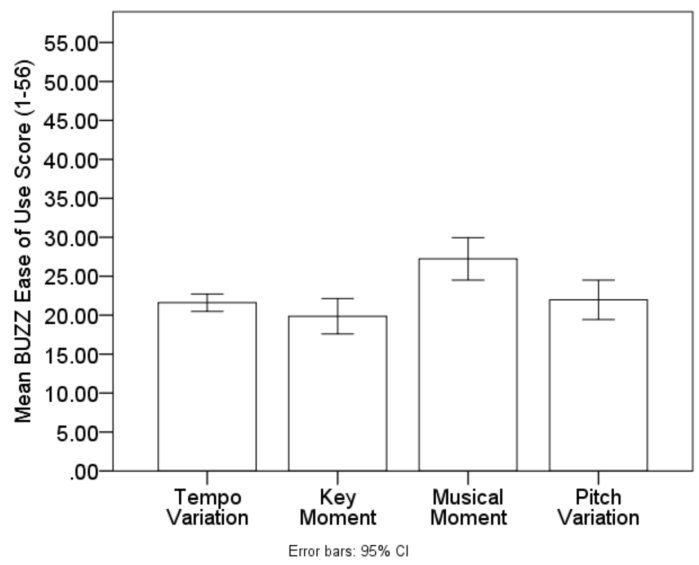

Figure 6: BUZZ Ease of Use Scores (out of 56) by Sonification Method

\subsection{Sonification Methods for Mood}

Each sonification method we employed received different positives, and many commented on the mood created by each method. Musical moment alignment was commonly described as good for a highlight reel, but made the play feel as though it was not happening in real-time. Conversely, pitch mapping, tempo mapping and key moments made viewers feel much more involved in the play and as if the play was in real time.

\subsection{Creating for Entertainment}

There are many unique challenges when creating sonifications for entertainment and sport. The balance between entertainment and analysis is significant and emerged in both the quantitative and qualitative evaluations. The importance of each factor can be expected to vary between each viewer; levels of sonic information that could be distracting to some viewers might be considered insightful by others. There was a general consensus that the more information that was conveyed through sound, the less like music the sonification sounded. For some this meant a less enjoyable experience, while for others this enhanced their understanding of the play and their overall enjoyment in watching the play. In addition to participant discussions, the presence of this type of enjoyabilityusability trade-off was also shown through the BUZZ scores. Although the Pitch Sonification condition exhibited the highest overall BUZZ scores, analyses of BUZZ sub-scales revealed that this advantage came from the fact that participants found this version to be the most enjoyable, even though they rated it as less usable than the Musical Moment condition. This indicates the presence of a trade-off between enjoyability and usability in these two higherperforming auditory display approaches. 


\section{CONCLUSION}

Through a divergent creation process we established four methods of sonification that can be used for soccer. After evaluation, each method showed varied strengths and weaknesses, however we had the most positive overall response to Key Moments and Pitch Mapping, with the former being more usable and the latter being more enjoyable. While we developed multiple strategies for sonification and lessons learned from evaluation, there are still many open questions and new potential strategies applied. Going forward, a key step will be to evaluate interactive user control over sonification choices and how this impacts user experience. Ultimately, we have demonstrated the potential for improved viewer experience through soccer sonification.

\section{ACKNOWLEDGEMENTS}

We are grateful to Steve Gera and Dave Anderson from the GAINS Group for discussions and suggestions during the beginning stages of this project, and for encouraging us to keep moving ahead on this project to make sports more accessible through the use of technology.

\section{REFERENCES}

[1] C. Carling, J. Bloomfield, L. Nelsen, and T. Reilly, "The role of motion analysis in elite soccer," Sports medicine, vol. 38, no. 10, pp. 839-862, 2008.

[2] E. Rampinini, F. M. Impellizzeri, C. Castagna, A. J. Coutts, and U. Wisløff, "Technical performance during soccer matches of the italian serie a league: Effect of fatigue and competitive level," Journal of science and medicine in sport, vol. 12, no. 1, pp. 227-233, 2009.

[3] T. Strudwick and T. Reilly, "Work-rate profiles of elite premier league football players," Insight, vol. 2, no. 2, pp. 28 29, 2001

[4] G. Vigne, A. Dellal, C. Gaudino, K. Chamari, I. Rogowski, G. Alloatti, P. Del Wong, A. Owen, and C. Hautier, "Physical outcome in a successful italian serie a soccer team over three consecutive seasons," The Journal of Strength \& Conditioning Research, vol. 27, no. 5, pp. 1400-1406, 2013.

[5] S. Barrass, N. Schaffert, and T. Barrass, "Probing preferences between six designs of interactive sonifications for recreational sports, health and fitness," Proceedings of ISon, pp. $23-29,2010$

[6] J. W. Newbold, N. Bianchi-Berthouze, and N. E. Gold, "Musical expectancy in squat sonification for people who struggle with physical activity." Georgia Institute of Technology, 2017

[7] G. Schmitz and A. O. Effenberg, "Perceptual effects of auditory information about own and other movements." Georgia Institute of Technology, 2012.

[8] O. Höner, T. Hermann, and C. Grunow, "Sonification of group behavior for analysis and training of sports tactics," in in Proc. of the International Workshop on Interactive Sonification, Bielefeld, 2004.

[9] N. Schaffert, K. Mattes, and A. O. Effenberg, "A sound design for the purposes of movement optimisation in elite sport (using the example of rowing)." Georgia Institute of Technology, 2009.

[10] B. Stahl and B. Thoshkahna, "Real-time heart rate sonification for athletes." Georgia Institute of Technology, 2015.

[11] N. Schaffert, K. Mattes, S. Barrass, and A. O. Effenberg, "Exploring function and aesthetics in sonifications for elite sports," in Proceedings of the 2nd international conference on music communication science (ICoMCS2), vol. 83. HCSNet, 2009, p. 86.

[12] T. Hermann and S. Zehe, "Sonified aerobics-interactive sonification of coordinated body movements." International Community for Auditory Display, 2011.

[13] T. Stockman, N. Rajgor, O. Metatla, and L. Harrar, "The design of interactive audio soccer." Georgia Institute of Technology, 2007.

[14] S. A. Pettersen, D. Johansen, H. D. Johansen, V. BergJohansen, V. Reddy, A. Mortensen, R. Langseth, C. Griwodz, H. K. Stensland, and P. Halvorsen, "Soccer video and player position dataset," in MMSys, 2014.

[15] R. Savery and G. Weinberg, "Shimon the robot film composer and deepscore," in Computer Simulation of Musical Creativity, 2018.

[16] R. Savery, "An interactive algorithmic music system for edm," Dancecult: Journal of Electronic Dance Music Culture, vol. 10, no. 1, 2018.

[17] R. J. Savery, "Algorithmic improvisers," Master's thesis, 2015.

[18] B. J. Tomlinson, B. E. Noah, and B. N. Walker, "Buzz: An auditory interface user experience scale," in Extended Abstracts of the 2018 CHI Conference on Human Factors in Computing Systems, ser. CHI EA '18. New York, NY, USA: ACM, 2018, pp. LBW096:1-LBW096:6. [Online]. Available: http://doi.acm.org/10.1145/3170427.3188659 